TechnoLEARN: An International Journal of Educational Technology

TechnoLEARN: 9(2): 77-82, December 2019

DOI: $10.30954 / 2231-4105.02 .2019 .3$

(C2019 New Delhi Publishers. All rights reserved

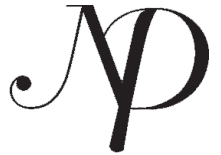

\title{
Technology-Enhanced Student-Centered Learning Environments
}

\section{Neeta Pandey}

A.T. P.S. Bhiti-1, Handia, Prayagraj, Uttar Pradesh, India

Corresponding author: neetaparthsarathi@gmail.com

\begin{abstract}
Technology-enhanced, student-centered learning environments organize interrelated learning themes into meaningful contexts, often in the form of a problem to be solved or an orienting goal, that bind functionally their features and activities. They provide interactive, complimentary activities that enable individuals to address unique learning interests and needs, study multiple levels of complexity, and deepen understanding. They establish conditions that enrich thinking and learning, and use technology to enable flexible methods through which the processes can be supported. Many technology-enhanced student-centered learning environments have been developed, ranging from situated, problem-based approaches, to micro worlds, to specialized manipulation tools. Research on these environments, while promising, has focused largely on the presumed uniqueness of the approaches. Among constructivists, beliefs about how to promote understanding vary widely. Design guidelines and heuristics have occasionally been offered, but they have not stimulated what Glaser (1976) characterized as a "science of design." Consequently, apart from isolated studies, comparatively little understanding of the role of technology in the design of student centered learning environments has evolved. The purposes of this paper are to provide a brief overview of technology-enhanced, student-centered learning environments, and to identify the foundations and underlying assumptions common across studentcentered designs.
\end{abstract}

Keywords: Student-Centered Learning, Learning Environments, Technology

\section{The Emergence of Technology in Student-Centered Learning Environments}

Learning systems of enormous power and sophistication have been developed to represent evolving notions of the partnerships among learners, experience, discourse, and knowledge. Student-centered learning systems reflect research and theory ranging from situated, contextual teaching and learning to resource-based models of education. 
Y)

Pandey

\section{Views about learning}

Student-centered learning environments evolved as a result of shifting beliefs and assumptions about the role of the individual in learning. Contemporary designers have been influenced heavily by constructivists who assert that understanding transcends the encoding of literal information and is uniquely constructed (Guba, 1990; Jonassen, 1991; Phillips, 1995). Knowledge must be assimilated; perceptions of value, meaning, and importance must be tentatively derived; existing knowledge must be evaluated concurrently with new knowledge; and understandings must be reconstructed accordingly. In effect, student-centered learning environments emphasize constructing personal meaning by relating new knowledge to existing conceptions and understandings; technology promotes access to resources and tools that facilitate construction. Recently, researchers have examined how learners evolve understanding in technology-rich learning environments. Effective environments support the individual's intentions to derive and solve problems through the use of available resources and tools. The result is a complex interaction among prior knowledge, perception of events, intents, actions, observations, and reflections attendant to on-going thoughts and actions. Actions, goals, and processes are initiated as a result of both previous system experiences and intuitive assumptions about the concepts under study. Learning, then, is a dynamic process of "reflection-in-action" where action is used to extend thinking, and reflection is governed by the results of action.

\section{Views About Teaching}

The focus has often been on developing critical thinking, problem solving, and reasoning skills. The overarching goals are to encourage manipulation rather than simple acquisition, and to root the learning process in concrete experience. These systems, it has been argued, represent fundamentally different views and beliefs about teaching and the nature of learning, not a simple re-hosting of traditional approaches. The utility of instructional approaches, the means through which traditional teaching and learning assumptions are often operationalized, has been detailed by several theorists who have derived very different inferences noted that instruction must focus on broader, more integrative outcomes than typically assumed, a theme that has become increasingly popular. Merrill, Li, \& Jones cited the closed nature of traditional approaches, absence of guidance for interaction design, and limited adaptation as constraints of traditional models of computer-based learning. They advocated an extension of traditional models to account for the capabilities of emerging technologies. While Merrill and his colleagues advocate changes in the systems used to generate instruction, their underlying assumptions as to the nature of learning remains consistent with objectivist epistemology. Increased interest in student-centered learning has been evident. Yet, the nature of the systems seems, to many, to be more dissimilar than alike. It is important to recognize both similarities and differences among technology enhanced, student-centered environments. 


\section{Technology}

Simplified use has increased interest in classroom applications of "learning by designing". Technology has been harnessed to accomplish conventional aims, but comparatively few applications have unleashed the potential of either the technologies or learners. Studentcentered learning environments represent significant potential for optimizing the capabilities of both technology and learners. Rapid developments in technology have influenced the evolution of student centered learning environments. Complex information systems can now be designed and accessed for individual purposes with comparative ease. Emerging information systems, such as the World Wide Web, support varied student-centered approaches in a variety of settings. Integrated multimedia platforms are now commonplace, providing powerful systems for developing and using highly sophisticated learning environments. Software innovations have also been prominent. Significant advances in authoring, multimedia development, production tools, simulation software, and expert system shells have been apparent. Software developments have increased not only the power and versatility of emerging systems, but have made them increasingly friendly and intuitive. Individuals can uniquely define the purposes of technology's uses, and exploit its capabilities to support individual interests and needs. Teaching-learning approaches have often been re-hosted, not re-defined. Improved understanding of the foundations and assumptions of such systems is needed.

\section{Foundations of technology-enhanced student-centered learning environments}

Learning environments are rooted in five foundations: Psychological, pedagogical, technological, cultural, and pragmatic. Direct instruction environments typically draw upon foundations that are consistent with objectivist, designer centered perspective. Student-centered learning environments' foundations, on the other hand, reflect a more user-centered view about the nature of knowledge and the role of the learner. Both are rooted in psychological foundations, but the approaches differ.

\section{Psychological}

Technology-enhanced, student-centered learning environments manifest diverse psychological foundations. All learning environments, explicitly or tacitly, reflect underlying beliefs about how knowledge is acquired and used. Psychological foundations reflect views about how individuals acquire, organize, and deploy knowledge and skill. Psychological foundations

are subsequently operationalized through various design frameworks, activities, and strategies, which reflect beliefs about how individuals think, learn, understand, and act. Historically, learning environments were rooted psychologically in behaviorism, with stimulusresponse-reinforcement associationism as the core explanatory learning paradigm. Relevant information was presented, practice elicited, and specific, contiguous feedback provided. Thus, 
\%

Pandey

understanding is derived through experience. Ideally, student-centered learning environments emphasize concrete experiences that serve as catalysts for constructing individual meaning. Despite apparent variations, however, common psychological foundations are manifested in the roles of technology in supporting activities, features, and opportunities to support student-initiated, student-directed understanding.

\section{Pedagogical}

Pedagogical influences focus on the activities, methods, and structures of the learning environment; pedagogical foundations emphasize how an environment is designed and its affordances are made available. In concert with an underlying psychological model, they provide the basis for the methods and strategies employed and the ways in which to-be-learned content is organized. Pedagogical foundations represent the operational bases for the different methods and activities generated using varied design models. Direct instruction approaches frequently emphasize instructional strategies such as hierarchical structure of to-be learned content, objective-relevant questioning, feedback, and assessment of progress toward mastery. In contrast, generative activities such as learning strategy training and learner choice and control are designed to capitalize on the unique cognitive capabilities of individual learners. While external structure tends to influence the success of such strategies, they are designed to empower the learner with methods that are widely applicable across diverse learning tasks. In each case, the pedagogical options reflect distinctly different underlying assumptions, and draw upon different pedagogical foundations. Technology-enhanced, student-centered learning environments establish contexts that promote sampling, discovering, manipulating, and investigating.

The individual must reason before acting, assess what needs to be understood, and identify and execute methods believed helpful. Using technological tools, students navigate, reference on-line resources, conduct experiments, and collect data in their quest for a solution. They need to reason before acting, assess their needs, identify and select methods believed helpful, and reflect on the information selected, encountered, generated, or constructed (Land \& Hannafin, in press).

Technology-enhanced, student-centered learning environments create contexts within which knowledge and skill are authentically anchored, and provide a range of tools and resources with which to navigate and manipulate. They afford opportunities to seek rather than to comply, to experiment rather than to accept, to evaluate rather than to accumulate, and to interpret rather than to adopt. Yet, they may also draw upon related constructs, such as generative strategies and elaboration. Pedagogical foundations, therefore, are not confined to methods derived from constructivism, but represent a synthesis of research and theory which establishes contexts, resources, and tools to promote learning. 


\section{Technological}

Technology-enhanced, student-centered learning environments often facilitate understanding of abstract concepts via concrete experience. For instance, a thermodynamics environment allows learners to collect real-time temperatures of various objects, noting changes as they are displayed graphically. Taken independently, technological capabilities suggest what is possible through advances in technology, not necessarily what is required or desired. When considered with the other foundations, technological foundations represent how the capabilities and limitations of available technologies can be optimized. Technologies can be distinguished by the operations they support and the symbol systems they employ. Technological foundations influence the design of learning systems by establishing the toolkit available to both the designer and the learner. Computer scan monitor responses, provide individualized feedback about choices, and maintain records of performance. However, these capabilities exist independently of particular design assumptions or decisions; design decisions regulate how, or if, technological capabilities will be utilized. Technological capabilities dictate not how much learner control is supported, but how much is possible. They determine not what should be, but what could be. Learners vary parameters such as initial temperature, surface area, and insulation material. Technological tools, in this instance, redefine the experiences available to learners and the cognitive requirements of a learning task. Technological capabilities may also promote heretofore untested designs and strategies. They can redefine what is possible or feasible and stimulate new perspectives on the teaching-learning process. The challenge for designers is to capitalize on the capabilities of emerging technologies based upon existing designs, while generating new designs rooted in emerging psychological and pedagogical research and theory. For such shifts to occur, foundations related to teaching, learning and technology, and the features related to those foundations, need to be aligned.

\section{Cultural}

Cultural foundations reflect prevailing beliefs about education, the values of a culture, and the roles of individuals in society. More recently, the need to meet the knowledge requirements of our rapidly expanding technological society has emerged. Computers are increasingly prevalent in classrooms and educational software is widely available; schools mirror the values and priorities of an increasingly technological society. Cultural foundations influence the design of learning systems by reflecting social mores and values concerning the nature and role of education. They provide intensive classroom instruction, administer highly competitive national tests to determine eligibility for very select colleges and universities, and require significant commitments by families to ensure the futures of children. Evolution in a given culture's educational priorities occur because of the real need to increase, decrease, or shift focus based upon prevailing attitudes, beliefs, and societal mores. The same can be said for 
P

Pandey

individual school districts, schools, classrooms, teachers, instructional units, and classroom modules. Each reflects, in a very real sense, the philosophy of its parent organization (e.g., school boards, teachers). This is important in any learning system, but it is of special relevance in the design of learning environments. The culture affecting learning environments can be traced to groups such as scientists, engineers, corporations, and advocacy groups.

\section{Pragmatic}

Pragmatic foundations bridge the gap between theory and reality. They emphasize the practical reasons a particular approach can or cannot be used in a given learning environment. Pragmatics might also dictate that learning environments blend aspects of varied pedagogical models. In a very real sense, pragmatic foundations dictate what can be in a learning environment, accounting for both human and technological assets and limitations as well as situational factors. As technological, psychological, and pedagogical research and theory continues to advance, designers must develop systems that accommodate the real constraints of the learning environment while overcoming those rooted in narrowness of their perspectives.

\section{An integrated view}

Though presented in isolation, the foundations are functionally integrated in learning system designs. The various manners in which they are manifested reflect fundamentally different assumptions about the nature of teaching, learning, knowing, and understanding.

\section{REFERENCES}

Bacon, S. 1987. The evolution of the Outward Bound process. Greenwich, GT: Outward Bound USA.

Bell, M. 1993. What constitutes experience? Rethinking theoretical assumptions. Journal of Experiential Education, 16(1): 19-24.

Menand, L. 2001. The metaphysical club. New York: Farrar, Straus and Giroux.

Priest, S. 1996. The effect of two different debriefing approaches on developing self-confidence. Journal of Experiential Education, 19(6): 40-42.

Sugerman, D.A., Doherty, K.L., Garvey, D.E. and Gass, M.A. 2000. Reflective learning: Theory and practice. Dubuque, IA: Kendall/Hunt.

Vokey, D. 1987. Outward Bound: In search of foundations. Unpublished doctoral dissertation. Queen's University, Kingston, Ontario, Canada. 